\title{
A Decoupling Based Direct Method for Power System Transient Stability Analysis
}

\author{
Bin Wang, Kai Sun \\ Electrical Engineering and Computer Science \\ University of Tennessee, Knoxville, TN USA \\ bwang13@utk.edu, kaisun@utk.edu
}

\author{
Xiaowen $\mathrm{Su}$ \\ Mechanical, Aerospace and Biomedical Engineering \\ University of Tennessee, Knoxville, TN USA \\ xsu2@utk.edu
}

\begin{abstract}
This paper proposes a decoupling based direct method to analyze the post-contingency transient stability for a general multi-machine power system. A linear decoupling transformation is used to construct the same number of independent single machine infinite bus (SMIB) power systems as oscillation modes. Each SMIB system carries stability information regarding one oscillation mode of the original system at the equilibrium. Then, the transient energy function method is applied on all decoupled SMIB systems to calculate stability margins, the smallest of which indicates the stability margin of the original system. Case studies on an IEEE 9-bus power system and a WECC 179-bus power system demonstrate the validity of the proposed method.
\end{abstract}

Index Terms - transient stability, transient energy function, direct method, linear decoupling, decoupled systems, oscillation mode, contingency screening.

\section{INTRODUCTION}

Dynamic security assessment (DSA) programs are important for modern power systems to operate stably and securely under more stressed conditions due to the increasing demand in electricity markets and penetration of intermittent resources. Fast contingency screening techniques play an important role in online DSA programs for identification of the most critical contingencies from a given list and direct methods are one of the effective means employed in fast contingency screening to quickly access the stability level for each post-contingency condition so as to rank these contingencies. Many direct methods have been proposed along with the performance indices during the last several decades, which may vary from system model, indices formulation and thresholds selection. These differences largely determine their reliability and efficiency.

The ways how a power system may lose its angular stability have strong relationships with its oscillation modes [1]. A multi-machine power system may have a large number of oscillation modes, either inter-area or local. Those modes couple, together influence dynamics of generators, and largely increase the complexity in transient stability analysis. Thus, understanding and even decoupling those oscillation modes may enable more efficient transient stability analysis.

In the realm of mechanical engineering, the total decoupling of general linear second-order differential equations by a

This work was supported by the University of Tennessee in Knoxville and the CURENT Engineering Research Center. real-valued linear transformation becomes available [2][3]. It transforms the equations of a linear system into a collection of mutually independent equations such that each equation can be solved without solving any other equation. However, very few studies on decoupling have been made for power systems [4][5], where the efforts were mainly put on the signal processing and the mechanism of decoupling is still not clear. In power systems, linearization based analysis usually adopts the state space representation in small signal analysis. Another representation based on the synchronizing coefficients is also capable to do such analysis [6]. The latter one is used in this paper which provides a novel perspective for system analysis.

An assumption is used throughout the paper without mathematical proof: a multi-machine power system can be decoupled into a number of independent single-machine-infinite-bus (SMIB) power systems. This assumption implies that all power system nonlinearities are considered by the decoupled SMIB systems and the modal interaction between any two modes is supposed to be zero. However, it is difficult to analytically derive a universal transformation used for complete decoupling of all on- and off-equilibrium system states into the states of those fictitious SMIB systems. Fortunately, a necessary condition of those SMIB systems, if they could exist, would be to coincide with a group of systems linearly decoupled from the linearized model of the original power system around the equilibrium point. As shown later in this paper, the parameters of that group of linear systems are found sufficient to determine those SMIB systems. However, existence of a universal transformation has not been proved. As an initial step of research in this direction, this paper tests a linear transformation of system states for identifying parameters of the aforementioned group of decoupled linear systems as well as of fictitious SMIB systems. The term decoupling method used in this paper describes the process to obtain those fictitious SMIB power systems.

The decoupled SMIB systems are supposed to represent the oscillatory behavior of the original power system. If any of these SMIB power systems is unstable, the original system will be considered unstable. Thus, checking the stabilities of the decoupled SMIB systems is equivalent to checking the stability of the original system. The transient energy function based direct method is adopted to estimate the stability margin of each decoupled SMIB system, where the smallest margin is chosen 
as the stability margin of the original system. When using the direct method, the initial system states during the post-fault period need to be transformed into the decoupled coordinates. Since the universal transformation for off-equilibrium states is currently unavailable, the proposed linear transformation is used as a compromise in this step.

Section II introduces the decoupling method and section III proposes a direct method based on those decoupled SMIB power systems. Case studies on the IEEE 9-bus system and the WECC 179-bus system are presented in section IV and conclusions are provided in section $\mathrm{V}$.

\section{Derivation OF DECOUPLing METHOD}

\section{A. Derivation of linear decoupling transformation}

Consider a general $m$-machine power system

$\ddot{\delta}_{i}=\frac{\omega_{0}}{2 H_{i}}\left(P_{m, i}-E_{i}^{2} G_{i}-\sum_{j=1, j \neq i}^{m}\left(C_{i j} \sin \delta_{i j}+D_{i j} \cos \delta_{i j}\right)\right)$

where $i \in\{1,2, \ldots, m\}, \delta_{i j}$ represents the rotor angle difference between machine $i$ and machine $j, \delta_{i}, P_{m, i}$, and $E_{i}$ represent the absolute rotor angle, mechanical power and field voltage of machine $i$, respectively, and $G_{i}, C_{i j}$ and $D_{i j}$ represent network parameters including loads, which are modeled by constant impedances.

Assume that the system is operating at its equilibrium at $t=0 \mathrm{~s}$ and there is no disturbance. Since all rotor angles increase at a common angular speed $\omega_{0}$, the absolute rotor angle of machine $i$, say $\delta_{i}^{s}$, can be calculated by (2).

$$
\delta_{i}^{s}=\delta_{i}^{s 0}+\omega_{0} t
$$

where $\delta_{i}^{s 0}$ is the initial absolute rotor angle of machine $i$.

The angle differences at the steady-state can be obtained.

$$
\delta_{i j}^{s}=\delta_{i}^{s}-\delta_{j}^{s}=\delta_{i}^{s 0}-\delta_{j}^{s 0}
$$

where $i, j \in\{1,2, \ldots, m\}$ and $i \neq j$.

The linearization of (1) at the system equilibrium could be obtained by (4) and (5).

$$
\begin{gathered}
\ddot{\delta}_{i}+a_{i 0}+\sum_{j=1}^{m} a_{i j} \delta_{j}=0 \\
\left\{\begin{array}{l}
a_{i j}=\frac{\omega_{0}}{2 H_{i}}\left(D_{i j} \sin \delta_{i j}^{s}-C_{i j} \cos \delta_{i j}^{s}\right) \quad \text { for } \quad j \neq i \\
a_{i i}=-\sum_{j=1, j \neq i}^{m} a_{i j} \\
a_{i 0}=\sum_{j=1, j \neq i}^{m} a_{i j} \delta_{i j}^{s}
\end{array}\right.
\end{gathered}
$$

Let $\boldsymbol{A}=\left\{a_{i j}\right\}_{m \times m}, \boldsymbol{\Omega}$ be a diagonal matrix whose diagonal elements are $\boldsymbol{A}$ 's eigenvalues and $\boldsymbol{U}$ be a matrix whose columns are $\boldsymbol{A}$ 's eigenvectors corresponding to the eigenvalues in $\boldsymbol{\Omega}$. Then the linear decoupling transformation is defined by (6) and new coordinates of the linearly decoupled system are defined by (7).

$$
\boldsymbol{T}=\boldsymbol{U}^{-1}=\left(\begin{array}{cccc}
\tau_{1,1} & \tau_{1,2} & \cdots & \tau_{1, m} \\
\tau_{2,1} & \tau_{2,2} & \cdots & \tau_{2, m} \\
\vdots & \vdots & \ddots & \vdots \\
\tau_{m, 1} & \tau_{m, 2} & \cdots & \tau_{m, m}
\end{array}\right)
$$

$$
\left[q_{1}, q_{2}, \cdots, q_{m}\right]^{T}=\boldsymbol{T}\left[\delta_{1}, \delta_{2}, \cdots, \delta_{m}\right]^{T}
$$

For further analysis, two properties of $\boldsymbol{A}$ and $\boldsymbol{T}$ are used, whose proof can be found in [6]. Property 1: matrix $\boldsymbol{A}$ has one zero eigenvalue and $(m-1)$ positive eigenvalues. Property 2: the sum of elements in each row of $\boldsymbol{T}$, corresponding to a non-zero eigenvalue, is zero.

The zero eigenvalue of $\boldsymbol{A}$ represents a component in the motion of all rotors which can change freely without any constraint. This motion corresponds to the fact that all rotor angles increase or decrease collectively in any stable power system, which is also called the "mean motion" of all machines in [6]. The other $(m-1)$ eigenvalues represent the $(m-1)$ natural oscillation frequencies at the equilibrium. Assume that the zero eigenvalue is the last element of $\boldsymbol{\Omega}$.

Note that for any eigenvector in $\boldsymbol{U}$, when multiplied by any non-zero scalar, it will still be an eigenvector corresponding to the same eigenvalue. Thus, the ambiguity of $\boldsymbol{U}$ results in the fact that $\boldsymbol{T}$ is not definite by (6). To avoid the ambiguity and gain more physical meaning, $\boldsymbol{T}$ is normalized such that: i) for any of $\boldsymbol{T}$ 's first $(m-1)$ row vectors, the sum of its positive elements is 1 , then the sum of its negative elements is -1 by property 2 ; ii) for $\boldsymbol{T}$ 's last row vector, the sum of its elements is one. The transformation matrix $\boldsymbol{T}$ used in the rest of the paper is the one after this normalization.

In sum, a linear decoupling transformation $\boldsymbol{T}$ is proposed for the linearized model of a general $m$-machine power system. It can be used to completely decouple the linearized system shown in (4). Next subsection will show how to use $\boldsymbol{T}$ to decouple the original nonlinear system shown in (1).

\section{B. Derivation of decoupled SMIB power systems}

By assumption, an $m$-machine power system could be completely decoupled into $(m-1)$ mutually independent fictitious SMIB power systems by a certain transformation $\boldsymbol{T}_{c}$, which would be time-variant, nonlinear or both. Each decoupled SMIB power system has the form shown in (8) with two unknown parameters $q_{k 0}$ and $\beta_{k}$, where $k \in\{1,2, \cdots, m-1\}$ [9]. Note that at the equilibrium, $\boldsymbol{T}_{c}$ should be the same as the linear decoupling transformation $\boldsymbol{T}$, such that (6) and (7) also hold. Based on the steady-state condition, two connections between each fictitious SMIB system and the original system shown in (1) can help determine the unknown parameters $q_{k 0}$ and $\beta_{k}$. One connection is from the steady-state value of rotor angles according to (7) and the other one is from the natural frequency, which are shown in (9) and (10), respectively. It can be concluded that if the $m$-machine power system could be completely decoupled into many fictitious SMIB power systems, these fictitious systems should be uniquely determined by (8), (9) and (10) based on $\boldsymbol{T}$ and $\boldsymbol{\Omega}$.

$$
\begin{gathered}
\ddot{q}_{k}+\beta_{k}\left(\sin \left(q_{k}+q_{k 0}\right)-\sin q_{k 0}\right)=0 \\
q_{k 0}=\sum_{j=1}^{m} \tau_{k j} \delta_{j}^{s} \\
\beta_{k} \cos q_{k 0}=\omega_{k}^{2} \Longrightarrow \beta_{k}=\frac{\omega_{k}^{2}}{\cos q_{k 0}}
\end{gathered}
$$




\section{Discussions}

The above synchronizing coefficients based analysis is the same as the small signal analysis when the system can be expressed by a set of second-order differential motion equations, i.e. classical models for generators, since they are based on the linearization of the same nonlinear system. In this case, the former one is computationally less intensive because the size of the related matrix is half of that in small signal analysis.

It should be emphasized that the linear transformation $\boldsymbol{T}$ is achievable as long as the linearization $\boldsymbol{A}$ of the nonlinear system is diagonalizable. Thus, the linear transformation can be achieved for cases with weak resonance while it cannot be achieved for cases with strong resonance [8]. This paper only considers cases whose linearizations are diagonalizable.

When calculating $\boldsymbol{T}$, the system equilibrium is required. However, without time-domain simulation for a long enough period, the post-fault steady-state is usually unknown before reached. Compromises could be made by estimating the postfault steady-state in ways which can avoid time-domain simulation. In this paper, a compromise is made by replacing the post-fault steady-state by the pre-fault steady-state.

The universal transformation $\boldsymbol{T}_{c}$ used for completely decoupling the multi-machine power system can hardly be obtained and the acquisition of $\boldsymbol{T}_{c}$ should be at least as difficult as the proof of the decoupability of a general multi-machine power system. However, at the system equilibrium, $\boldsymbol{T}_{c}$ should be identical to the linear transformation $\boldsymbol{T}$, which can be easily calculated. Note that using $\boldsymbol{T}$ to decouple the system states of the original system is only valid at the equilibrium. If using $\boldsymbol{T}$ to decouple system states away from the equilibrium, errors will inevitably be involved. To transform off-equilibrium system states, more nonlinearities should be considered to estimate a better transformation such as the one in [7] which considers the nonlinearity of the system up to the second order.

\section{PROPOSED DIRECT METHOD BASED ON DECOUPLING}

Consider a SMIB system shown in (11)

$$
\Delta \ddot{\delta}+\frac{P_{\max } \omega_{0}}{2 H}\left(\sin \left(\delta_{0}+\Delta \delta\right)-\sin \delta_{0}\right)=0
$$

where $\Delta \delta$ is the rotor angle deviation relative to its postfault steady-state value $\delta_{0}, P_{\max }$ is the steady-state maximum power transfer, $\omega_{0}$ is the synchronous frequency and $H$ represents the inertia of the machine.

A fault is added to the system and cleared after a certain time. Assume two initial values at the fault clearing time to be $\Delta \delta(0)$ and $\Delta \dot{\delta}(0)$. The objective of a direct method is to estimate the stability of the system using those values without proceeding with the time-domain simulation and give an index for stability margin.

Based on the transient energy function method, the critical energy for this SMIB power system is defined by (12) and the sum of the system's kinetic and potential energies at the fault clearing time is define by (13) and (14). A normalized energy margin index [10] is usually defined by (15).

$$
V_{c r}=P_{\max }\left(2 \cos \delta_{0}-\left(\pi-2 \delta_{0} \sin \delta_{0}\right)\right)
$$

$$
\begin{gathered}
V_{c}=V_{k c}+V_{p c} \\
\left\{\begin{array}{l}
V_{k c}=\frac{H}{\omega_{0}}(\Delta \dot{\delta}(0))^{2} \\
\left.V_{p c}=P_{\max }\left(\cos \delta_{0}-\cos \Delta \delta(0)-\left(\Delta \delta(0)-\delta_{0}\right) \sin \delta_{0}\right)\right)
\end{array}\right. \\
\Delta V_{n}=\frac{V_{c r}-V_{c}}{V_{k c}}=\frac{2 V_{c r} / P_{\max }-2 V_{c} / P_{\max }}{2 V_{k c} / P_{\max }}
\end{gathered}
$$

The system is claimed to be stable when $V_{c r}>V_{c}$ or $\Delta V_{n}>0$. Otherwise, the system is unstable. Since $P_{\max }$ and $H$ are not defined for each decoupled SMIB system such that (12), (13) and (14) cannot be directly calculated. But (15) can still be calculated according to its definition.

An ideal direct method based on the decoupling should use the post-fault steady-state to obtain the decoupled SMIB systems and use $\boldsymbol{T}_{c}$ to transform the initial values from the original system states into the decoupled coordinates. But the universal $\boldsymbol{T}_{c}$ is currently unavailable and the postfault steady-state may not be known accurately without timedomain simulation. Thus, two approximate direct methods are proposed using the linear decoupling transformation $T$ as below: 1) direct method I (DM-I) uses post-fault steady-state estimated from a short period of time-domain simulation; 2) direct method II (DM-II) uses pre-fault steady-state.

\section{Case studies}

The classical generator model and the constant impedance load model are used for all simulations in this paper.

\section{A. Tests on IEEE 9-bus power system}

The first test uses both DM-I and DM-II. A three-phase fault is added on the line 4-5 near bus 4 at $t=1$ second and cleared after a certain time by tripping the line 4-5. The critical clearing time (CCT) identified by a number of simulation runs is 0.197 second. Fig. 2 and Fig. 3 show a stable case with fault clearing time $T_{c}=0.19 \mathrm{~s}$ and an unstable case with $T_{c}=0.20 \mathrm{~s}$, respectively. In each of the two cases, two oscillation modes are excited which are $0.8 \mathrm{~Hz}$ and $1.7 \mathrm{~Hz}$. Based on the proposed direct methods, $2 V_{c r} / P_{\max }, 2 V_{c} / P_{\max }$ and $\Delta V_{n}$ for each mode with different $T_{c}$ are calculated and shown in Tables. I and II, respectively. The results from DMI and DM-II are almost the same since all machines are in classical models without exciters and governors such that its equilibrium with the line tripped is basically the same. In these cases, DM-II is faster than DM-I with no significant loss of accuracy. In addition, DM-II can give a severity index by $\Delta V_{n}$ for unstable cases while DM-I cannot since there is no postfault steady-state if the system is unstable.

The second test uses DM-II to rank all line-tripping contingencies. For each contingency, a three-phase fault is added at one end of the line and cleared after $0.1 \mathrm{~s}$. Feeding DMII with the initial values of the post-fault period, the normalized energy margin is calculated for each contingency. To demonstrate the ranking result, the CCT of each contingency is also provided in the last column of Table.III. In this result, most critical contingencies identified by DM-II roughly match those with smallest CCTs. It shows that the normalized energy margin from DM-II could be a stability index of the system. 


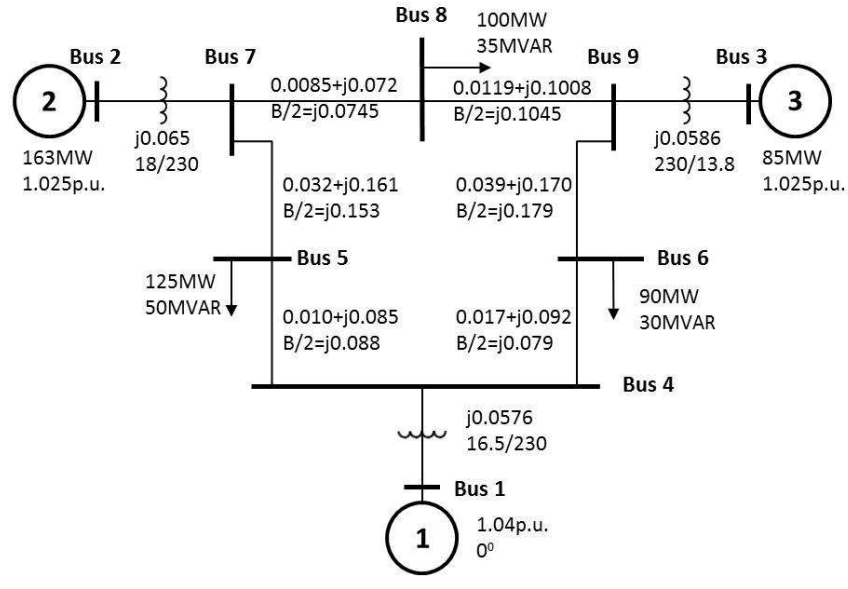

Fig. 1. IEEE 9-bus power system
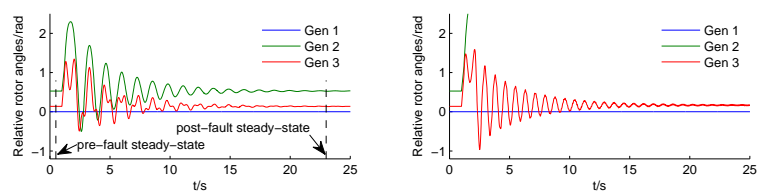

Fig. 2. Relative angles $\left(T_{c}=0.19 s\right)$ Fig. 3. Relative angles $\left(T_{c}=0.20 s\right)$

TABLE I

RESULTS ON $0.8 \mathrm{HZ}$ MODE WITH DIFFERENT $T_{c}$

\begin{tabular}{c|ccc|ccc}
\hline \multirow{2}{*}{$T_{c} / \mathrm{s}$} & \multicolumn{3}{|c|}{ DM-I } & \multicolumn{3}{c}{ DM-II } \\
\cline { 2 - 7 } & $\frac{2 V_{c r}}{P_{\max }}$ & $\frac{2 V_{c}}{P_{\max }}$ & $\Delta V_{n}$ & $\frac{2 V_{c r}}{P_{\max }}$ & $\frac{2 V_{c}}{P_{\max }}$ & $\Delta V_{n}$ \\
\hline 0.01 & 21.0 & 0.05 & 445 & 21.0 & 0.05 & 445 \\
0.05 & 21.0 & 1.17 & 17.2 & 21.0 & 1.17 & 17.2 \\
0.09 & 21.0 & 3.87 & 4.65 & 21.0 & 3.87 & 4.65 \\
0.13 & 21.0 & 8.35 & 1.67 & 21.0 & 8.35 & 1.67 \\
0.17 & 21.0 & 14.9 & 0.47 & 21.0 & 14.9 & 0.47 \\
0.19 & 21.0 & 19.1 & 0.12 & 21.0 & 19.1 & 0.12 \\
0.20 & - & - & - & 21.0 & 21.5 & -0.03 \\
0.21 & - & - & - & 21.0 & 24.0 & -0.15 \\
\hline
\end{tabular}

TABLE II

RESULTS ON $1.7 \mathrm{HZ}$ MODE WITH DIFFERENT $T_{c}$

\begin{tabular}{c|ccc|ccc}
\hline \multirow{2}{*}{$T_{c} / \mathrm{s}$} & \multicolumn{3}{|c|}{ DM-I } & \multicolumn{3}{|c}{ DM-II } \\
\cline { 2 - 7 } & $\frac{2 V_{c r}}{P_{\max }}$ & $\frac{2 V_{c}}{P_{\max }}$ & $\Delta V_{n}$ & $\frac{2 V_{c r}}{P_{\max }}$ & $\frac{2 V_{c}}{P_{\max }}$ & $\Delta V_{n}$ \\
\hline 0.01 & 185 & 0.03 & $7 \mathrm{e} 4$ & 185 & 0.03 & $7 \mathrm{e} 4$ \\
0.05 & 185 & 0.69 & 288 & 185 & 0.69 & 288 \\
0.09 & 185 & 2.48 & 92.1 & 185 & 2.48 & 92.1 \\
0.13 & 185 & 6.00 & 46.0 & 185 & 6.00 & 46.0 \\
0.17 & 185 & 12.1 & 28.0 & 185 & 12.1 & 28.0 \\
0.19 & 185 & 16.4 & 22.9 & 185 & 16.4 & 22.9 \\
0.20 & - & - & - & 185 & 18.9 & 20.8 \\
0.21 & - & - & - & 185 & 21.7 & 19.0 \\
\hline
\end{tabular}

The third test uses DM-II to deal with a case with two similar modes. In order to obtain such case, the power flow is re-dispatched following [11] and the inertias of generators 1,2 and 3 are changed to 7.8, 6 and 15, respectively. By such modifications, two oscillatory modes become $1.03 \mathrm{~Hz}$ and $1 \mathrm{~Hz}$. When adding a temporary three-phase fault on the line 4-5 near bus 4 , the CCT is identified to be $0.327 \mathrm{~s}$. Table.IV shows the results from DM-II with different $T_{c}$. In this case, the normalized margin does not cross zero from the marginally stable to the unstable case. The $T_{c}$ of the marginally stable case predicted by DM-II is as large as $0.43 \mathrm{~s}$. Consequently, it can be concluded that the decoupling based DM-II is unable to provide an accurate margin for cases with two similar modes.

\section{B. Tests on WECC 179-bus power system}

A simplified 29-machine 179-bus model of the WECC power system is used to test the proposed DM-II. As shown in Fig.4, the shaded area is a subsystem to add contingencies. Consider all $N-1$ single-line-tripping contingencies in that subsystem. Each of them follows a 5-cycle three-phase-fault at one end of a line. The top-15 critical contingencies are chosen to test the proposed DM-II. Time-domain simulation shows that eight of them are unstable and the rest seven are stable. The normalized energy margins from DM-II are shown in Table.V where the CCTs are also provided in the last column. It can be seen that all unstable contingencies are captured by DM-II while two stable contingencies are selected as unstable. The results are conservative since the proposed direct method ignores the effect from damping at each machine

TABLE III

RANKING RESULT OF LINE-TRIPPING CONTINGENCIES BY DM-II

\begin{tabular}{cc|cc|cc}
\hline $\begin{array}{c}\text { Faulted } \\
\text { Line }\end{array}$ & $\begin{array}{c}\text { Fault } \\
\text { Near bus }\end{array}$ & $\begin{array}{c}\text { Ranking } \\
\text { by } \Delta V_{n}\end{array}$ & $\Delta V_{n}$ & $\begin{array}{c}\text { CCT } \\
\text { Ranking }\end{array}$ & $\begin{array}{c}\text { CCT } \\
\text { /s }\end{array}$ \\
\hline $5-7$ & 7 & 1 & 1.61 & 5 & 0.174 \\
$8-9$ & 8 & 2 & 1.77 & 1 & 0.139 \\
$7-8$ & 7 & 3 & 2.00 & 2 & 0.156 \\
$6-9$ & 9 & 4 & 3.13 & 4 & 0.172 \\
$7-8$ & 8 & 5 & 3.14 & 6 & 0.184 \\
$8-9$ & 9 & 6 & 3.42 & 3 & 0.169 \\
$4-5$ & 4 & 7 & 3.57 & 7 & 0.197 \\
$4-5$ & 5 & 8 & 3.70 & 9 & 0.212 \\
$5-7$ & 5 & 9 & 3.88 & 11 & 0.229 \\
$4-6$ & 4 & 10 & 4.61 & 8 & 0.201 \\
$6-9$ & 6 & 11 & 5.96 & 10 & 0.221 \\
$4-6$ & 6 & 12 & 6.46 & 12 & 0.231 \\
\hline
\end{tabular}

TABLE IV

RESULTS ON TWO SIMILAR MODES WITH DIFFERENT $T_{c}$

\begin{tabular}{c|ccc|ccc}
\hline \multirow{2}{*}{$T_{c} / \mathrm{s}$} & \multicolumn{3}{|c|}{$1.03 \mathrm{~Hz}$ mode } & \multicolumn{3}{c}{ 1Hz mode } \\
\cline { 2 - 7 } & $\frac{2 V_{c r}}{P_{\max }}$ & $\frac{2 V_{c}}{P_{\max }}$ & $\Delta V_{n}$ & $\frac{2 V_{c r}}{P_{\max }}$ & $\frac{2 V_{c}}{P_{\max }}$ & $\Delta V_{n}$ \\
\hline 0.01 & 57.8 & 0.02 & $3 \mathrm{e} 3$ & 77.5 & 0.02 & $4 \mathrm{e} 3$ \\
0.10 & 57.8 & 2.21 & 27.9 & 77.5 & 1.78 & 46.9 \\
0.20 & 57.8 & 9.67 & 7.25 & 77.5 & 7.63 & 13.3 \\
0.30 & 57.8 & 24.5 & 2.76 & 77.5 & 19.0 & 6.53 \\
0.32 & 57.8 & 28.6 & 2.21 & 77.5 & 22.0 & 5.76 \\
0.33 & 57.8 & 30.7 & 1.96 & 77.5 & 23.7 & 5.41 \\
0.43 & 57.8 & 57.2 & 0.03 & 77.5 & 44.2 & 2.47 \\
0.44 & 57.8 & 60.3 & -0.11 & 77.5 & 46.7 & 2.22 \\
\hline
\end{tabular}




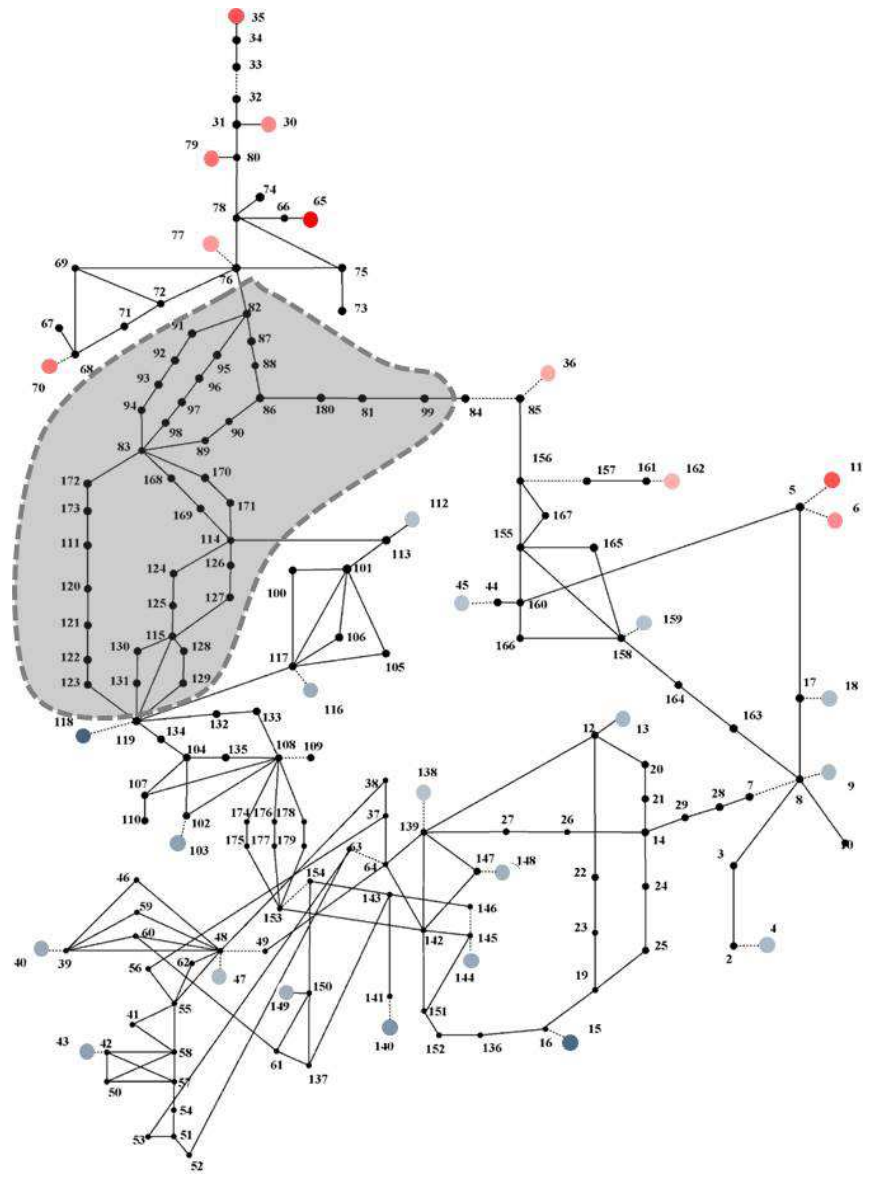

Fig. 4. WECC 179-bus power system

when calculating the normalized energy margin.

In addition, 28 oscillation modes are found: 7 modes with frequencies less than $1 \mathrm{~Hz}, 11$ modes between $1 \mathrm{~Hz}$ and $2 \mathrm{~Hz}$ and 10 modes larger than $2 \mathrm{~Hz}$. The $0.43 \mathrm{~Hz}$ mode is found to be the dominant one in each of those 15 critical contingencies and two other modes, $0.56 \mathrm{~Hz}$ and $2.2 \mathrm{~Hz}$, could have fairly small margins for some contingencies while still larger than that of $0.43 \mathrm{~Hz}$ mode. The mode shape of the $0.43 \mathrm{~Hz}$ is also provided in Fig.4, where machines denoted by solid red circles are oscillating against those in solid blue. How much each machine is involved is expressed by the shade of the color.

\section{CONCLUSIONS}

This paper assumes that a general multi-machine power system can be completely decoupled into many independent SMIB power systems, where all the nonlinearities of the original system are considered by the nonlinearities of all SMIB systems. A linear transformation is used to derive these SMIB systems and the transient energy function based direct method is applied to each of them to assess the transient stability of the original system. To provide the initial system states for the direct method, the linear transformation is used as a compromise of the universal decoupling transformation. Case studies on the IEEE 9-bus system and the WECC 179- bus system show that the proposed decoupling based direct method has a potential to be used for fast transient stability analysis or contingency screening in power system dynamic security assessment.

The investigations of the assumption used in this paper, better transformations for decoupling off-equilibrium system states and better estimates of post-fault system steady-state are problems for further research, where the resonance phenomenon and the nonlinear modal interaction should also be considered.

\section{REFERENCES}

[1] K. Sun, X. Luo, J. Wong, "Early Warning of Wide-Area Angular Stability Problems Using Synchrophasors," IEEE PESGM, San Diego, CA, Jul. 2012

[2] M.T. Chu and N.D. Buono, "Total decoupling of general quadratic pencils, part i: theory," J. of Sound and Vibration, 309(1-2), pp.96-111, Dec. 2008

[3] M. Morzfeld, "The transformation of second-order linear systems into independent equations,'Ph.D. dissertation, Dept. Mechanical Eng., Univ. of California, Berkeley, Spring, 2011

[4] C. Zhang and G. Ledwich, "A new approach to identify modes of the power system based on T-matrix," Sixth International Conference on ASDCOM, Hong Kong, Nov. 2003

[5] G. Ledwich, "Decoupling for improved modal estimation," IEEE PES General Meeting, Tampa, FL, Jul. 2007

[6] F. Saccomanno, "Electromechanical phenomena in a multimachine system," in Electric Power Systems, New York: Wiley, 2003, pp.619-635

[7] R.J. Betancourt, E. Barocio, I. Martinez, A.R. Messina, "Modal analysis of inter-area oscillation using the theory of normal modes," Electric Power System Research, vol.79, no.4, pp.576-585, Apr. 2009

[8] K.R. Padiyar and H.V. SaiKumar, " Investigations on Strong Resonance in Multimachine Power Systems With STATCOM Supplementary Modulation Controller,' IEEE Trans. on Power Systems, vol.21, no.2, pp.754762, May 2006

[9] B. Wang, K. Sun, D.R. Alberto, E. Farantatos and N. Bhatt, "A study on fluctuations in electromechanical oscillation frequencies of power systems," IEEE PES General Meeting, National Harbor, MD, Jul. 2014

[10] H.D. Chiang, F.F. Wu, P.P. Varaiya, "A BCU method for direct analysis of power system transient stability," IEEE Trans. on Power Systems, vol.9, no.3, pp.1194-1208, Aug, 1994

[11] I. Dobson, J. Zhang, S. Greene, H. Engdahl, P.W. Sauer, "Is strong modal resonance a precursor to power system oscillations," IEEE Trans. on Circuits and Systems, vol.48, no.3, pp.340-349, Mar. 2001

TABLE V

RANKING RESULT OF LINE-TRIPPING CONTINGENCIES BY DM-II

\begin{tabular}{cc|cc|cc}
\hline $\begin{array}{c}\text { Faulted } \\
\text { Line }\end{array}$ & $\begin{array}{c}\text { Fault } \\
\text { Near bus }\end{array}$ & $\begin{array}{c}\text { Ranking } \\
\text { by } \Delta V_{n}\end{array}$ & $\Delta V_{n}$ & $\begin{array}{c}\text { Ranking } \\
\text { By CCT }\end{array}$ & $\begin{array}{c}\text { CCT } \\
\text { /s }\end{array}$ \\
\hline $130-131$ & 131 & 1 & -0.9520 & 6 & 0.049 \\
$119-131$ & 131 & 2 & -0.9519 & 6 & 0.049 \\
$115-130$ & 130 & 3 & -0.9014 & 1 & 0.030 \\
$130-131$ & 130 & 4 & -0.9009 & 1 & 0.030 \\
$87-88$ & 88 & 5 & -0.6886 & 3 & 0.035 \\
$86-88$ & 88 & 6 & -0.6874 & 3 & 0.035 \\
$170-171$ & 171 & 7 & -0.1212 & 5 & 0.048 \\
$168-169$ & 169 & 8 & -0.0665 & 6 & 0.049 \\
$81-180$ & 81 & 9 & -0.0476 & 9 & 0.104 \\
$81-99$ & 81 & 10 & -0.0441 & 9 & 0.104 \\
$86-180$ & 86 & 11 & 1.336 & 11 & 0.131 \\
$81-180$ & 180 & 12 & 26.13 & 12 & 0.892 \\
$86-180$ & 180 & 13 & 26.18 & 13 & 0.891 \\
$84-99$ & 99 & 14 & 75.69 & 14 & 2.45 \\
$81-99$ & 99 & 15 & 75.94 & 15 & 2.45 \\
\hline
\end{tabular}

